\title{
Non-thermal, Non-equilibrium, Indirect, Jet Plasma Treatment with Nitrogen Gas as a Therapeutic Option for Acne Vulgaris: A Case Report of Clinical Experiences
}

\author{
Hyoung-Moon Kim, MD ${ }^{1}$ \\ Min-Seung Kim, MD² \\ Ji-Sun Kim, MD ${ }^{3}$ \\ Wook Oh, $\mathrm{MD}^{4}$ \\ ${ }^{1}$ Maylin Clinic, Goyang, Korea \\ ${ }^{2}$ INEE Clinic, Seoul, Korea \\ ${ }^{3} \mathrm{MH}$ Clinic, Seoul, Korea \\ ${ }^{4}$ Maylin Clinic, the Hyundai, Seoul, Korea
}

Received October 8, 2021

Accepted October 11, 2021

\section{Correspondence}

Hyoung-Moon Kim

Maylin Clinic, Goyang 10391, Korea

E-mail: drmac12@me.com

https://orcid.org/0000-0001-9068-5813

(C) Korean Society for Laser, Dermatology and Trichology

(a) This is an open access article distributed under the terms of the Creative Commons Attribution NonCommercial License (http://creativecommons.org/ licenses/by-nc/4.0) which permits unrestricted noncommercial use, distribution, and reproduction in any medium, provided the original work is properly cited.

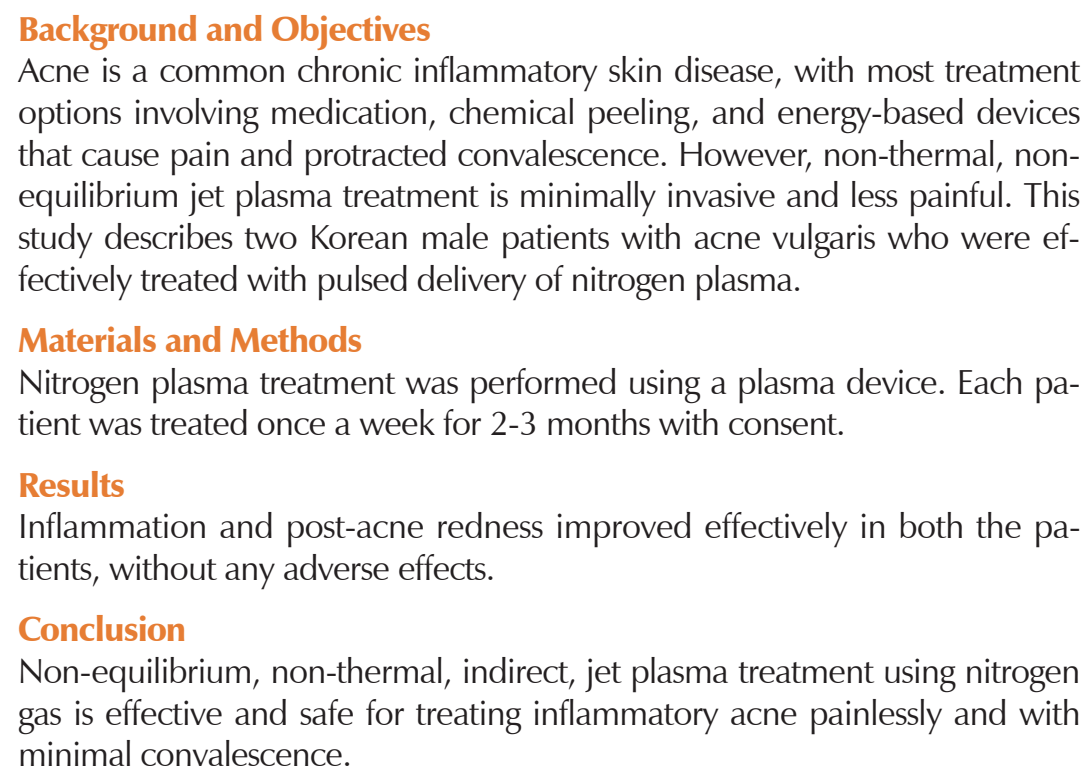

Acne is a common chronic inflammatory skin disease, with most treatment options involving medication, chemical peeling, and energy-based devices that cause pain and protracted convalescence. However, non-thermal, nonequilibrium jet plasma treatment is minimally invasive and less painful. This study describes two Korean male patients with acne vulgaris who were effectively treated with pulsed delivery of nitrogen plasma.

Materials and Methods

Nitrogen plasma treatment was performed using a plasma device. Each patient was treated once a week for 2-3 months with consent.

Results

Inflammation and post-acne redness improved effectively in both the patients, without any adverse effects.

\section{Conclusion}

Non-equilibrium, non-thermal, indirect, jet plasma treatment using nitrogen gas is effective and safe for treating inflammatory acne painlessly and with minimal convalescence.

\section{Key words}

Acne, Plasma, Nitrogen, Acne vulgaris, Jet plasma 


\section{INTRODUCTION}

Acne is a chronic inflammatory skin disease with known multifactorial causes. The main etiologies include increased sebum secretion, Cutibacterium (previously Propionibacterium) acnes growth, dermal inflammation, abnormal follicular keratinocyte proliferation, and desquamation [1-3]. Acne treatment involves oral and topical retinoids, antibiotic medication, chemical peeling, and energy-based modalities, such as sebum-specific wavelength laser or light and radiofrequency devices [4-7].

Plasma, the fourth state of matter, comprises charged electrons and ions, nitric oxide, and hydroxyl radicals. Nitrogen plasma has been clinically used for treatment of photodamaged skin and wrinkles by generating distinctive chromophore-independent energy-dependent zones of thermal damage and modification [8,9]. Plasma-induced cavitation (PIC) can be created in the epidermis and dermis. PIC increases collagen formation, which sometimes destroys melanin and sebaceous glands. PIC with high-energy settings enables cavitation in the more superficial epidermis, wherein it is referred as plasma shield phenomenon. In addition, high-energy nitrogen plasma setting can destroy sebaceous glands irreversibly with permanent scarring of the skin [10]. Our lower-energy parameter setting further ensures complete safety.

This study presents our clinical experience of lower-energy level nitrogen plasma treatment in two Korean patients with acne vulgaris and evaluates its effectiveness and safety.

\section{CASE REPORT}

\section{Case 1}

A 15-year-old Korean male patient visited our clinic and presented with erythematous papular lesions in the frontal portion, temple, nose, and lateral cheek and was diagnosed with cystic inflammatory acne. The patient had not been treated with any oral or topical medication or other peel- ing or laser treatment. He refused our suggestion of oral medication with isotretinoin because he wanted a less painful procedure. Therefore, we decided on nitrogen plasma treatment with comedo extraction. After obtaining written informed consent, the patient was treated by cleansing the face with a mild soap and extracting the comedones manually. We did not apply any chemical peels or other anesthetic cream. A single session of nitrogen plasma treatment was conducted using a plasma device (Pladuo ${ }^{\mathrm{TM}}$, ShenB Co., Ltd., Seoul, Korea) that generates plasma from argon and nitrogen gas sources emitted form microwave generator and delivers pulses to the skin surface. We used only nitrogen gas. Nitrogen plasma was prepared by loading $0.48 \mathrm{ml}$ nitrogen gas/pulse; a frequency of $2.45 \mathrm{GHz}$ was used to activate inert nitrogen gas. The other treatment settings included a pulse energy of $1.0 \mathrm{~J}$, pulse duration of $10 \mathrm{~ms}$, nozzle diameter of $5 \mathrm{~mm}$, and distance from the nozzle's tip to the skin of $10 \mathrm{~mm}$. Skin temperature varied by the distance from the nozzle to the skin surface (Fig. 1). The patient was treated for 3 months with 1-2-week intervals for a total of 10 sessions. After 3 months, he patient's inflammatory acne and post-acne erythema were markedly decreased (Fig. 2). Furthermore, skin texture and overall skin tone were improved, and the patient was very satisfied with the results. No remarkable major side effects, including itching, crusts, oozing, burning, worsening of inflammatory acne lesions or folliculitis, prolonged edema or erythema, or post-inflammatory hyperpigmentation, were noted.

\section{Case 2}

A 24-year-old male patient visited our clinic and presented with erythematous papular lesions on the temple and cheek. He had been treated with oral isotretinoin for improving the acne, but the medication was stopped due to severe dryness of the face. After obtaining written informed consent, the patient was managed with four sessions of nitrogen plasma treatment using a plasma device (Pladuo ${ }^{\mathrm{TM}}$ ) at 2-week intervals. Nitrogen plasma was prepared by load-
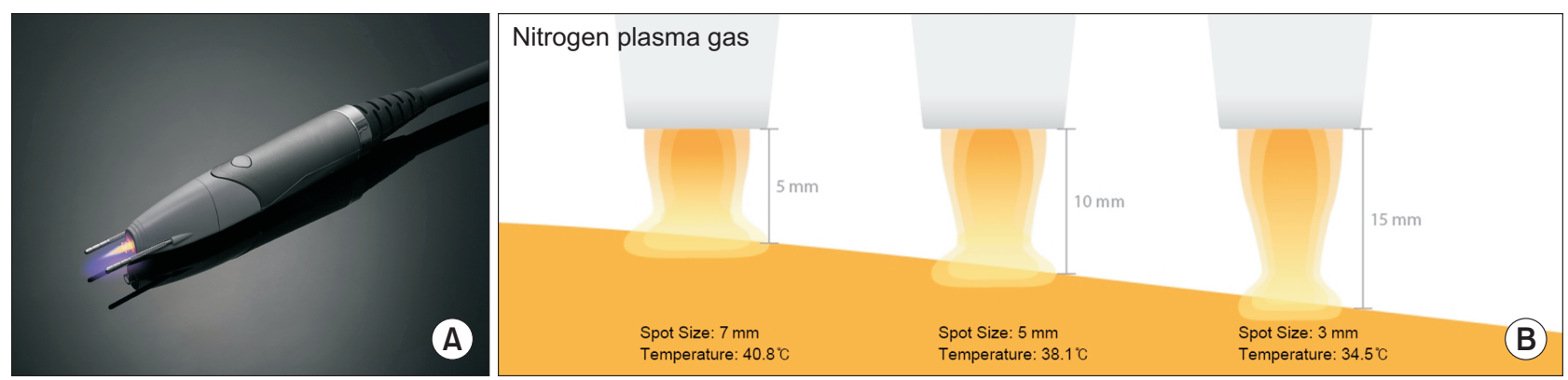

Fig. 1. (A) Pladuo ${ }^{\mathrm{TM}}$ handpiece with nitrogen gas jet plasma discharge. (B) Based on the distance from the nozzle to the skin surface, the temperature on the skin surface varies. Treatment settings included a pulse energy of $1.0 \mathrm{~J}$, pulse duration of $10 \mathrm{~ms}$, nozzle diameter of $5 \mathrm{~mm}$. 

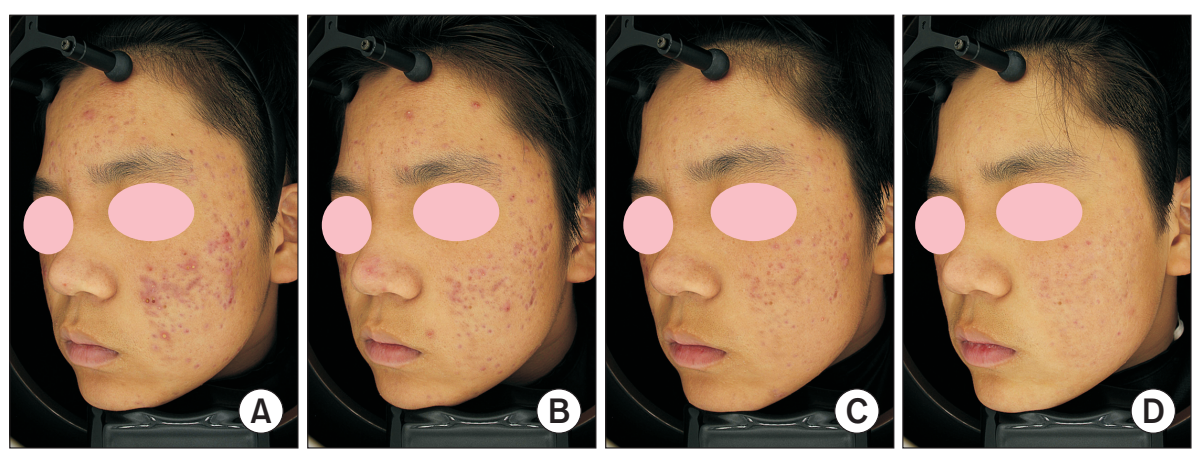

Fig. 2. Case 1. Photographs of a 15-yearold Korean male patient (A) at baseline, (B) at 1 month, (C) at 2 months, and (D) at 3 months after 10 sessions with a loading volume of $0.48-\mathrm{ml}$ nitrogen gas/ pulse, pulse energy of $1.0 \mathrm{~J}$, pulse duration of $10 \mathrm{~ms}$, nozzle diameter of $5 \mathrm{~mm}$, and distance from the nozzle's tip to the skin of $10 \mathrm{~mm}$. (A-D) Left oblique view.
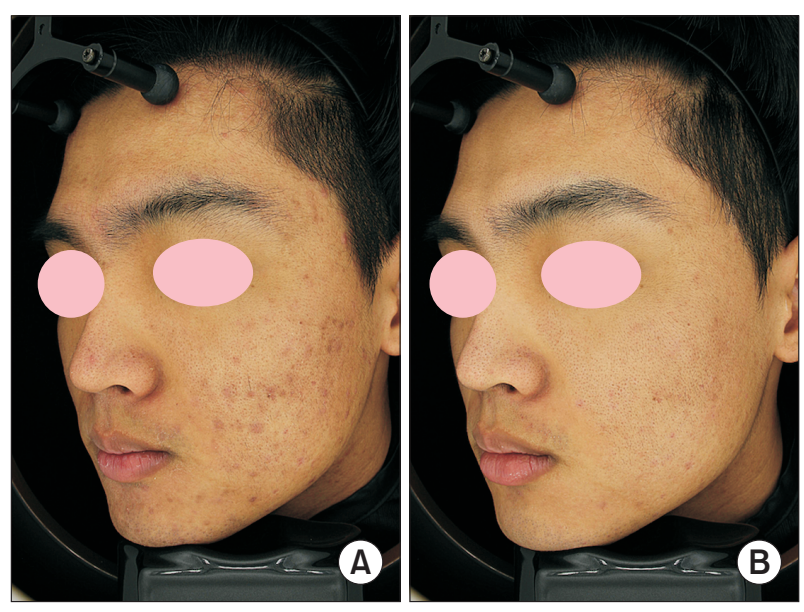

Fig. 3. Photographs of a 24-year-old Korean male patient (A) at baseline and (B) at 2 months after treatment. The patient was managed with four sessions of nitrogen plasma treatment at 2-week intervals with a pulse energy of $1.0 \mathrm{~J}$, pulse duration of $10 \mathrm{~ms}$, nozzle diameter of $5 \mathrm{~mm}$, and distance from nozzle's tip to the skin of $10 \mathrm{~mm}$. (A, B) Left oblique view.

ing $0.48 \mathrm{ml}$ nitrogen gas/pulse and was activated with a microwave generator. The other treatment settings included a pulse energy of $1.0 \mathrm{~J}$, pulse duration of $10 \mathrm{~ms}$, nozzle diameter of $5 \mathrm{~mm}$, and distance from nozzle's tip to the skin of 10 $\mathrm{mm}$. The patient's face was first cleansed with a mild soap and $70 \%$ ethanol. We performed 2-3 passes of nitrogen plasma treatment with a total shot count of approximately 800 shots/session, without any topical anesthetic agent. No prophylactic systemic antibiotics or chemical peeling were prescribed. The patient was recommended to avoid excessive ultraviolet (UV) radiation exposure. Four weeks after treatment, he exhibited marked reductions in inflammatory acne lesions, post-acne erythema, and hyperpigmentation (Fig. 3). Ultraviolet photographs taken at an identical setting using an imaging tool (Mark-Vu ${ }^{\circledR}$, PSIpuls, Seoul, Korea) revealed marked reductions in protoporphyrin IX by $C$. acnes (Fig. 4). Pore size, skin texture, and overall skin tone had improved, and the patient was very satisfied with the
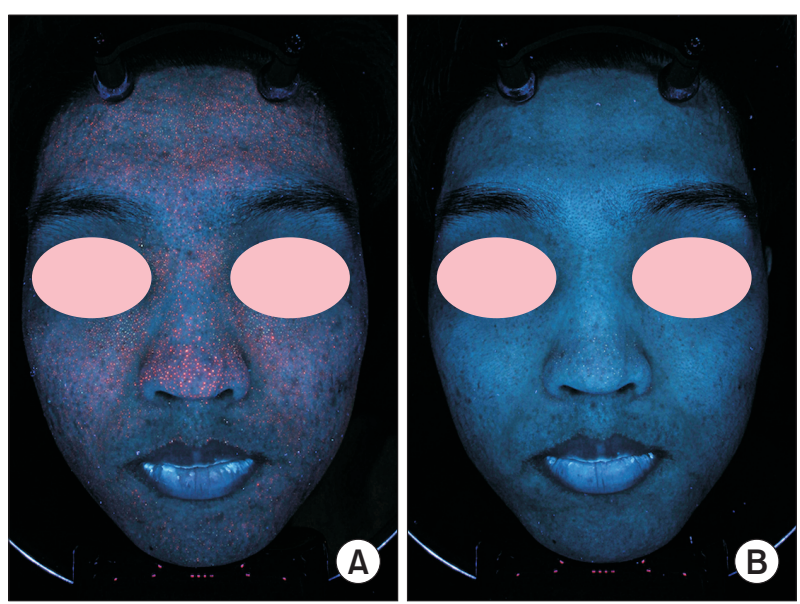

Fig. 4. Case 2. Photographs of a 24-year-old Korean female patient (A) at baseline and (B) 2 months after four sessions of nitrogen plasma treatment at 2-week intervals. Pinkish fluorescent areas in the centrofacial skin at baseline represent protoporphyrin IX by Cutibacterium. Photographs were taken under ultraviolet light exposure $(A, B)$.

results. Pain was tolerable without the use of a topical anesthetic cream, and most of the post-treatment erythema disappeared spontaneously within 2 hours of treatment. No remarkable major side effects were noted.

The two patients were satisfied after the plasma treatment sessions, especially in terms of improvement of inflammatory lesions with a less painful procedure. Acne has not recurred for approximately 3 months, and no other side effects were recorded.

\section{DISCUSSION}

Plasma devices are divided into thermal and nonthermal devices. Usually in medicine, we use non-thermal plasma that is classified into non-equilibrium and equilibrium plasma based on the equilibrium state between the temperature of electrons, ions, and neutral atoms. We commonly use non-thermal, non-equilibrium plasma, especially the lowest temperature band plasma $\left(20-60^{\circ} \mathrm{C}\right)$, that 
can be used for the treatment of living tissues without damage or with minimal damage to the surrounding healthy tissue $[7,8]$. The collisions of high-energy electrons with gas atoms or molecules result in generation of different reactive plasma species; the electrons can dissociate, ionize, or excite gas molecules. Following these electron-molecule reactions, ion-molecule reactions may also occur, resulting in different types of reactive species, especially reactive oxygen and nitrogen species [7].

Two main mechanisms of nitrogen plasma in living tissue are responsible for the UV-induced cellular damage at the molecular level: 1) direct effects based on UV energy absorption by cellular macromolecules and 2) alterations of DNA, proteins, and lipids caused by UV-induced oxidative stress. Moreover, non-thermal plasma does not cause an increased risk for genotoxicity [8,9].

Nitrogen plasma has also been used for various purposes due to its antibacterial, antifungal, and antiviral effects. It generates microscopic features of both thermal destruction and modification of pilosebaceous units depending on the energy setting and pulse number. In particular, treatment with high-energy settings over multiple passes generates extensive tissue coagulation in the epidermis, upper and mid-dermis, and sebaceous glands, eventually resulting in delayed wound repair and scarring [10].

In our study, we used low-level energy nitrogen plasma, with $1.0 \mathrm{~J}$ pulse energy, $10 \mathrm{~ms}$ pulse duration, and $5 \mathrm{~mm}$ nozzle diameter. Average total shots of $600-800$ to the entire face can induce mild erythema; however, it disappears approximately 2-3 hours later. We used the low-energy setting with nitrogen plasma gas to prevent scar formation and did not encounter any severe side effects. After high-energy setting nitrogen plasma irradiation, folliculitis, long-lasting erythema, burns, and scar formation may be experienced; therefore, we recommend caution with Pladuo ${ }^{\mathrm{TM}}$ above energy level 6 and suggest application of an anesthetic cream to the face above energy level 3 in Asian patients.

The advantage of plasma treatment for acne vulgaris is minimal pain without the use of an anesthetic cream, minimal down-time, and faster outcomes. Moreover, results such as improved skin texture and radiance can be obtained, which may be due to reduction in the epidermal hyperpigmentation [11].

In conclusion, our report suggests that non-thermal, non-equilibrium, indirect, jet plasma with low-energy nitrogen gas treatment for acne vulgaris is a safe and effective modality. Further investigations with a larger number of patients should be performed to elucidate the role of nitrogen plasma in acne treatment.

\section{ACKNOWLEDGEMENTS}

We would like to thank Sunny Kang (Shenb Co., Ltd., Seoul, Korea), Bora Kim (Shenb Co., Ltd.), and Min Choi (Shenb Co., Ltd.) for their technical assistance.

\section{CONFLICT OF INTEREST}

No potential conflict of interest relevant to this article was reported.

\section{REFERENCES}

1. Williams HC, Dellavalle RP, Garner S. Acne vulgaris. Lancet 2012;379:361-72.

2. Dréno B, Pécastaings $S$, Corvec $S$, Veraldi $S$, Khammari $A$, Roques $C$. Cutibacterium acnes (Propionibacterium acnes) and acne vulgaris: a brief look at the latest updates. J Eur Acad Dermatol Venereol 2018;32 Suppl 2:5-14.

3. Mayslich C, Grange PA, Dupin N. Cutibacterium acnes as an opportunistic pathogen: an update of its virulence-associated factors. Microorganisms 2021;9:303.

4. Elman M, Lebzelter J. Light therapy in the treatment of acne vulgaris. Dermatol Surg 2004;30(2 Pt 1):139-46.

5. Kurokawa I, Layton AM, Ogawa R. Updated treatment for acne: targeted therapy based on pathogenesis. Dermatol Ther (Heidelb) 2021;11:1129-39.

6. Li MK, Liu C, Hsu JTS. The use of lasers and light devices in acne management: an update. Am J Clin Dermatol. Forthcoming 2021. https://doi.org/10.1007/s40257-021-00624-5

7. Shukla SK, Gold MH. Treatment of acne and acne scars with microneedling. In: Houshmand EB, editor. Microneedling: Global Perspectives in Aesthetic Medicine. Hoboken: John Wiley \& Sons; 2021. p.81-97.

8. Heinlin J, Isbary G, Stolz W, Morfill G, Landthaler M, Shimizu T, et al. Plasma applications in medicine with a special focus on dermatology. J Eur Acad Dermatol Venereol 2011;25:1-11.

9. Heinlin J, Morfill G, Landthaler M, Stolz W, Isbary G, Zimmermann JL, et al. Plasma medicine: possible applications in dermatology. J Dtsch Dermatol Ges 2010;8:968-76.

10. Kim H, Kim HJ, Kim HK, Hong JY, Cho SB. Effects of argon and nitrogen plasma pulses on the skin and skin appendages in an in vivo animal model. Skin Res Technol 2020;26:81-90.

11. Kim HJ, Kim H, Kim YK, Cho SB. Treatment of refractory melasma with microwave-generated, atmospheric-pressure, non-thermal nitrogen plasma. Med Lasers 2019;8:74-9. 